\title{
Antiretroviral treatment for pregnant women living with HIV: A summary of issues, interventions, and evidence
}

Jill Gay

Melanie Croce-Galis

Karen Hardee

Population Council

Mackenzie Kacmarcik

\section{Recommended Citation}

Gay, Jill, Melanie Croce-Galis, Karen Hardee, and Mackenzie Kacmarcik. 2016. "Antiretroviral treatment for pregnant women living with HIV: A summary of issues, interventions, and evidence." Washington, DC:

Population Council, The Evidence Project and What Works Association. 


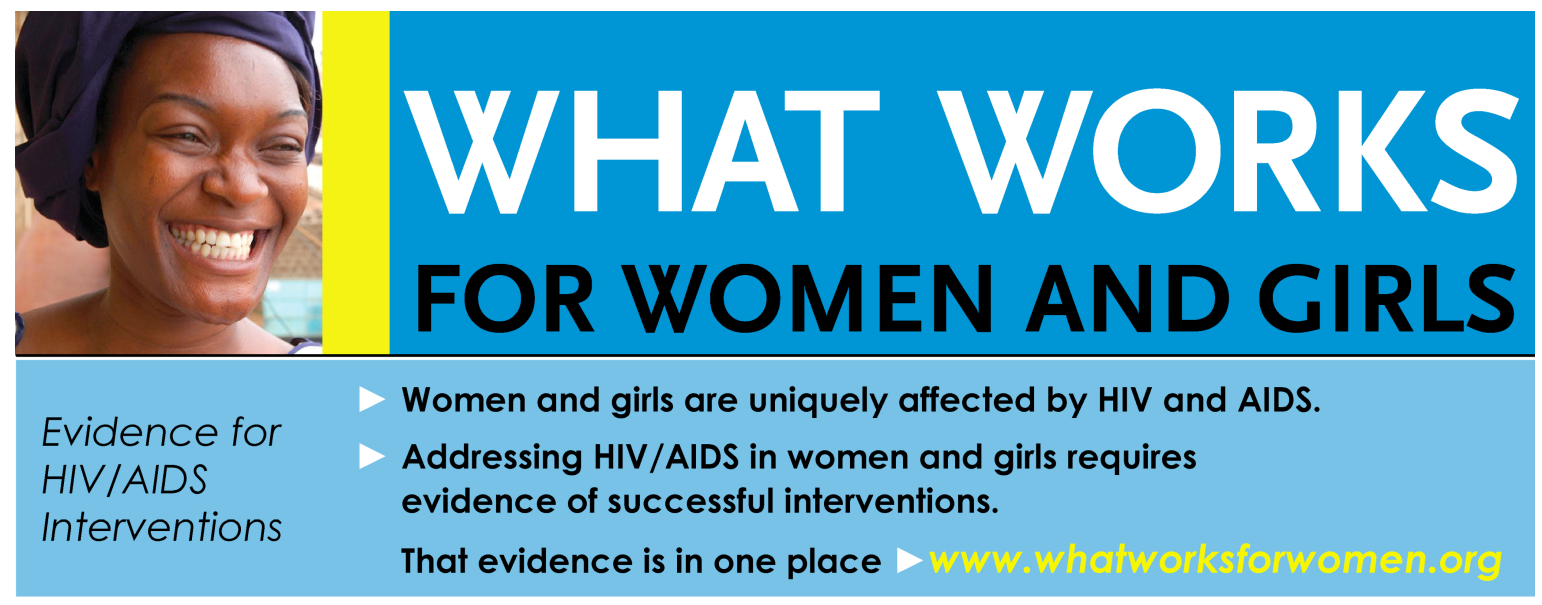

\section{Antiretroviral Treatment for Pregnant Women Living with HIV: A Summary of Issues, Interventions, and Evidence}

Antiretroviral treatment (ART) for women living with HIV is vital to ensuring safe motherhood and reducing vertical transmission. Each year, as many as 42,000 women living with HIV die of HIV and pregnancy-related complications (Glass and Birx, 2016). While significant progress has been made with $93 \%$ of pregnant women in 22 priority countries who have accessed combination ART (or cART, formerly called HAART), not all pregnant women can access treatment. In low- and middle-income countries in particular, treatment access for pregnant women living with HIV has been hampered by availability of medications and standardized treatment eligibility criteria that traditionally prioritized prevention of HIV transmission to the infant over treatment for the health of the woman.

\section{Treatment Guidance Has Changed Dramatically}

WHO's September 2015 guidance states that "ART should be initiated in all pregnant and breastfeeding women living with HIV at any CD4 cell count and continued lifelong," (WHO, 2015f: 13), a treatment regimen also known as Option B+. Global inequality may be reduced now that there is global agreement on when to start treatment. However, not all women will benefit immediately. The reality is

This section does not provide medical or clinical guidance, which is available from $\mathrm{WHO}$, but rather a public health perspective on what works for women in access and adherence to $A R T$ in the context of antenatal care. that, in 2015, fewer than one out of 10 people living with HIV live in a country where ART upon HIV diagnosis is current policy or practice (Health Gap, 2015).

Antiretroviral Medications are Beneficial, But Drug Resistance Remains a Concern for Women Living with HIV

Concerns about taking cART during pregnancy are outweighed by the benefits. Studies show that women living with HIV who access ART prior to pregnancy or very early in pregnancy have no differences in rates of birth defects in fetuses/infants with first trimester use of ART compared with ART initiation later in pregnancy (USHHS, 2015: c- 
6). Since Option $\mathrm{B}+$ calls for lifelong treatment, women living with HIV are at lesser risk of developing drug resistance, unless they stop and start the recommended treatment, are non-adherent, face drug stockouts, receive inappropriate regimens, etc.. Some women may face worse outcomes on cART due to earlier WHO-recommended regimens during previous pregnancies.

\section{All Women Living with HIV Need Timely Access to ART, Ideally Prior to Pregnancy} Adolescent girls are especially important in antenatal care treatment programming because of their increased vulnerability. Women who are part of key populations, such as women who use drugs and women who are sex workers, also need intensified programming to ensure ART access. "Motherhood is common among female sex workers," yet sex workers fear health services or avoid services, due to stigma and discrimination (Papworth et al, 2015; S154).

\section{Women's Lives Are as Important as Their Children's}

Maternal HIV status and health is key to survival for infants and children in the postneonatal period. A recent study in Malawi found that the mother's HIV-positive status correlated with more than one-third of deaths of children up to age four. Children whose mothers died were at greater risk of dying than those whose mothers were alive (Chihana et al., 2015). Focus group discussions among women living with HIV in Malawi found that Option $\mathrm{B}+$ is presented to women as a program primarily to protect the baby, with their health unimportant (Hsieh, 2013).

Few countries have comprehensive registers that follow the mother-infant pair after delivery to measure longer-term cART adherence (UNAIDS, 2015). In order to adequately assess if mothers are adhering well and are being kept alive, national governments should continue to track:

- What $\%$ of pregnant women living with HIV access ANC and cART (Dourado et al., 2014; Hlarlaithe et al., 2014);

- What \% of pregnant women living with HIV are virally suppressed with cART;

- What $\%$ of women living with HIV who remain virally suppressed with cART by number of years postpartum.

\section{Women Need Information, Support and Respect in Decision-Making About}

\section{Antiretroviral Therapy}

While WHO does not specify how quickly a pregnant woman should initiate cART upon an HIV-positive diagnosis, countries that are implementing Option $\mathrm{B}+$ are interpreting the guidance to mean immediately. Yet initiating treatment upon diagnosis may be too challenging for some pregnant women. Women in South Africa noted that they faced
"I was really in favour of early treatment and to have this Option $B+$. But now my worry is: are we being given this as an option or is this being pushed on us with no option?" -Woman living with HIV in Zimbabwe (ATHENA et al., 2015: 3).

a triple burden of transitioning into pregnancy, accepting a new HIV-positive serostatus and recognizing the need to start lifelong ART as soon as possible (Stinson and Myer, 2012). Insufficient counseling or 
respect for women's decision-making time can drive women away from accessing treatment.

Fear of Disclosure, Violence Can Influence Treatment Initiation and Adherence Pregnant women living with HIV still remain highly stigmatized in many countries. While "disclosure of one's HIV status can help to improve uptake and retention in prevention of mother-to-child transmission of HIV services..." (Tam et al., 2015: 436), some women are reluctant to disclose, particularly to their sexual partners (Croce-Galis et al., 2015). As one woman living with HIV, diagnosed during pregnancy, put it: “...I won't tell him, because I need him, because he helps me with money. I can't lose him now because I can't manage to have this baby if he doesn't support me" (Sewnunam and Modiba, 2015: 63). In some cases, disclosure may place a pregnant woman at risk. For example, pregnant women in Zimbabwe have faced violence for testing without their partner's consent (Shamu et al., 2014).

\section{Addressing Gender Norms and Supporting Women May be Key to Eliminating Vertical Transmission}

Women may face other gender related barriers to accessing health services (Croce-Galis et al., 2015), as one woman noted in Cote d'Ivoire, who said that her husband would not give her the funds to get transport to services (Schechter et al., 2014). A barrier to initiation, adherence and retention on ART for pregnant women living with HIV is that she may be required to ask permission to access services (Hodgson et al., 2014; Hlarlaithe et al., 2014). Key to gender transformative programming in Safe Motherhood and Prevention of Vertical Transmission is for women's lives to be valued - not just to keep babies healthy. Community-based support programs for pregnant women living with HIV can be helpful. A recent study of implementation of community-based adherence clubs for stable ART patients, which provided ART to 2,133 patients, 71\% female, with a strong emphasis on peer-based support and patient self management, found that one year later, only $6 \%$ of patients were lost to follow up and fewer than $2 \%$ of patients experienced viral load rebound (Grimsrud et al., 2015).

Additional Efforts are Needed to Better Engage Men in Supporting Safe Motherhood and Prevention of Vertical Transmission

Little work has been done to explain vertical transmission to male partners and how men can support pregnant partners living with HIV (Auvinen et al., 2014b). Interviews with male partners of pregnant women living with HIV in South Africa found that men felt responsible for their children, but that long clinic lines and the view that clinics for maternal health care are women-only spaces reduced the likelihood that men will access services through antenatal care (Koo et al., 2013a). Remarkably little is known about couples and their relationships in the context of HIV and how to improve couple communication around sensitive topics around risk, sex and transmission (RamirezFerrero and Lusti-Narasimhan, 2012). More nuanced efforts are needed to engage men in prevention of vertical transmission.

\section{It IS Possible to Eliminate Vertical Transmission}

Between 2009 and 2015, there has been a 46\% decline in the number of AIDS-related deaths among women of reproductive age in the 21 priority countries (UNAIDS, 2016), a 
remarkable achievement. In June 2015, Cuba became the first country to be validated as having met the global criteria for eliminating vertical transmission as a public health problem, that is, in 2014, fewer than 50 new infections in 100,000 live births; a rate of under $5 \%$ in breastfeeding women and less than $2 \%$ among women who do not breastfeed (WHO, 2015a, cited in UNAIDS, 2015; Gulland, 2015).

$* * *$

The following interventions have been successful in improving treatment for pregnant women living with HIV. See www.whatworksforwomen.org for more details, including full citations, detailed methodology, and critical gaps in the literature.

\section{What Works-Safe Motherhood and Prevention of Vertical Transmission: Antenatal Care - Treatment}

1. Initiating cART as early as possible to achieve low viral load is optimal, improves maternal health, and reduces risk of vertical transmission. Supported by 19 studies in 30 countries

2. Peer counseling by mother mentors may improve treatment adherence among pregnant women living with HIV. Supported by 6 studies in 8 countries

3. Community health workers and community-based support can increase uptake of safe motherhood interventions for women living with HIV and reduce vertical transmission. Supported by 9 studies in 7 countries

4. PMTCT-Plus (family-focused) HIV care can increase the numbers of women and their partners who access treatment and remain adherent. Supported by 4 studies in 9 countries

5. Integrating ARV therapy into antenatal care, rather than referring women separately for HIV treatment, can reduce time to treatment and increase adherence for pregnant women living with HIV. Supported by 10 studies in 5 countries

6. National scale-up of cART in pregnancy improves maternal and infant outcomes. Supported by 5 studies in 4 countries

What Works for Women and Girls is supported by the U.S. President's Emergency Plan for AIDS Relief (PEPFAR) and is carried out under the auspices of USAID's Evidence Project and What Works Association.

For more information please visit www.whatworksforwomen.org

Suggested Citation: Gay, Jill, Melanie Croce-Galis, Karen Hardee, and Mackenzie Kacmarcik. 2016. "Antiretroviral Treatment for Pregnant Women Living with HIV: A Summary of Issues, Interventions, and Evidence," Washington, DC: Population Council, The Evidence Project and What Works Association. 